TRANSACTIONS OF THE

AMERICAN MATHEMATICAL SOCIETY

Volume 355, Number 2, Pages 465-475

S 0002-9947(02)03173-2

Article electronically published on October 9, 2002

\title{
THE LAPLACIAN MASA IN A FREE GROUP FACTOR
}

\author{
ALLAN M. SINCLAIR AND ROGER R. SMITH
}

\begin{abstract}
The Laplacian (or radial) masa in a free group factor is generated by the sum of the generators and their inverses. We show that such a masa $\mathcal{B}$ is strongly singular and has Popa invariant $\delta(\mathcal{B})=1$. This is achieved by proving that the conditional expectation $\mathbb{E}_{\mathcal{B}}$ onto $\mathcal{B}$ is an asymptotic homomorphism. We also obtain similar results for the free product of discrete groups, each of which contains an element of infinite order.
\end{abstract}

\section{INTRODUCTION}

Dixmier introduced in 2 a classification of maximal abelian selfadjoint subalgebras (masas) $\mathcal{A}$ of a type $\mathrm{II}_{1}$ factor $\mathcal{M}$ in terms of the normalizing unitaries of $\mathcal{A}$. The von Neumann algebra generated by these unitaries could be the maximal case $\mathcal{M}$, the minimal case $\mathcal{A}$, or a factor containing $\mathcal{A}$ but strictly contained in $\mathcal{M}$. These three possibilities are respectively termed regular (or Cartan), singular and semi-regular. In many ways the masas in a von Neumann factor are the simplest subalgebras, and yet have a rich structure which can act as an isomorphism invariant. For example, many factors arising from the crossed product construction have canonical masas which are Cartan, while the factors arising from the free groups on $k$ generators do not, [11. In contrast to the regular masas, every factor has a singular masa, as shown by Popa, [4], and there are explicit examples in various group von Neumann factors, [1], 2], 4], 9], 10]. The purpose of this paper is to examine in some detail the Laplacian (or radial) masas in free group factors from the standpoint of a theory of strong singularity which we introduced in [10]. These masas arise naturally from nonabelian harmonic analysis on groups and play a significant role in the theory, [3], 8 .

It can be quite difficult to recognize the particular type of a given masa. We introduced in 10 the notion of strong singularity for masas, defined by a metric inequality which estimates the distance of a unitary $u$ from $\mathcal{A}$ in terms of the distance from $\mathcal{A}$ to $u \mathcal{A} u^{*}$ (see (2.3)). Such masas are singular, although the reverse implication is open at this time. The importance of this concept is that in many instances verification of singularity can be achieved without difficulty. The technique for this is based on the existence of asymptotic homomorphisms, which are conditional expectations with additional properties. These are discussed in the next section.

Received by the editors February 26, 2001 and, in revised form, July 26, 2002.

2000 Mathematics Subject Classification. Primary 46L10, 46L09.

The second author was partially supported by a grant from the National Science Foundation. 
Let $g_{1}, \ldots, g_{k}$ be the generators of the free group $\mathbb{F}_{k}, k \geq 2$, and let

$$
w_{1}=\sum_{i=1}^{k}\left(g_{i}+g_{i}^{-1}\right) \text {. }
$$

Then the Laplacian masa $\mathcal{B}$ is defined to be the abelian subalgebra of $V N\left(\mathbb{F}_{k}\right)$ generated by the selfadjoint element $w_{1}$. Pytlik, 8 , showed that $\mathcal{B}$ is indeed a masa, and subsequently Rădulescu, [9], proved that it is singular by establishing that the Pukánszky invariant, $[7$, is $\{\infty\}$. Popa, 5 , had already demonstrated that a masa with this particular Pukánszky invariant is singular. We will show that $\mathcal{B}$ is a strongly singular masa, and also that Popa's delta invariant, 4], has value 1 in this case. Since submission of the paper, $\delta(\mathcal{B})=1$ now follows from a much stronger result in [6]. As noted above, our route to establishing this is to show that $\mathbb{E}_{\mathcal{B}}$ is an asymptotic homomorphism, Theorem 5.1. since strong singularity and $\delta(\mathcal{B})=1$ are then implied by [10, Theorem 4.7]. We achieve this by proving a sufficient condition (Theorem 3.1), phrased in terms of the convergence of certain infinite series. Specifically, we need that the difference between $\mathbb{E}_{\mathcal{B}}\left(x w_{n} y\right)$ and $\mathbb{E}_{\mathcal{B}}(x) \mathbb{E}_{\mathcal{B}}(y) w_{n}$, where $w_{n}$ is the sum of words of length $n$ and $x$ and $y$ are fixed words in $\mathbb{F}_{k}$, should tend to 0 rapidly as $n \rightarrow \infty$. We also show, in Theorem 5.4 that our results extend to certain free products of groups (see [1] for similar work).

The second section contains background material and definitions of the terms mentioned above. The heart of the paper is the fourth section, in which we obtain various enumeration results for words in $\mathbb{F}_{k}$. These are used to show that the Laplacian masa satisfies the hypothesis of Theorem 3.1 from which strong singularity follows.

\section{Preliminaries}

In this section we present definitions and notation which will be needed subsequently. Specific notation about the number of words starting and ending in a certain way is contained in the fourth section, where it is used.

The operator norm on a type $\mathrm{II}_{1}$ factor $\mathcal{M}$ with normalized trace tr is denoted by $\|\cdot\|$, and $\|\cdot\|_{2}$ is the norm $\|x\|_{2}=\left(\operatorname{tr}\left(x^{*} x\right)\right)^{1 / 2}$. If $\mathcal{N}$ is a von Neumann subalgebra of $\mathcal{M}$, then $\mathbb{E}_{\mathcal{N}}$ denotes the trace-preserving conditional expectation of $\mathcal{M}$ onto $\mathcal{N}$. If $\phi: \mathcal{M}_{1} \rightarrow \mathcal{M}_{2}$ is a linear map between type $\mathrm{II}_{1}$ factors, then there are several norms on $\phi$, depending on the norms given to $\mathcal{M}_{1}$ and $\mathcal{M}_{2}$. We write $\|\phi\|$ and $\|\phi\|_{2}$ when both factors have respectively the operator norm and the $\|\cdot\|_{2}$-norm. When $\mathcal{M}_{1}$ has the operator norm and $\mathcal{M}_{2}$ has the $\|\cdot\|_{2}$-norm, we denote the resulting norm of $\phi$ by $\|\phi\|_{\infty, 2}$. This last norm was introduced and studied in [10]. We note that conditional expectations are contractions for all three norms.

A maximal abelian selfadjoint subalgebra (masa) in $\mathcal{M}$ is singular if any unitary $u \in \mathcal{M}$ which normalizes $\mathcal{A}\left(u \mathcal{A} u^{*}=\mathcal{A}\right)$ must lie in $\mathcal{A}$, [2]. A masa $\mathcal{A}$ has Popa invariant $\delta(\mathcal{A})=1$ if and only if the following is satisfied: for each nilpotent $\left(v^{2}=0\right)$ partial isometry $v \in \mathcal{M}$ with $v \mathcal{A} v^{*}$ and $v^{*} \mathcal{A} v$ contained in $\mathcal{A}$, we have

$$
\sup \left\{\left\|\left(I-\mathbb{E}_{\mathcal{A}}\right)(x)\right\|_{2}: \quad x \in v \mathcal{A} v^{*},\|x\| \leq 1\right\} \geq\left\|v v^{*}\right\|_{2},
$$

[4], 10]. In [10] we introduced the concept of strong singularity for masas $\mathcal{A}$. The defining property is

$$
\left\|u-\mathbb{E}_{\mathcal{A}}(u)\right\|_{2} \leq\left\|\mathbb{E}_{u \mathcal{A} u^{*}}-\mathbb{E}_{\mathcal{A}}\right\|_{\infty, 2}
$$


for all unitaries $u \in \mathcal{M}$. Singularity is clearly implied by (2.2), and we note that a reverse inequality

$$
\left\|\mathbb{E}_{u \mathcal{A} u^{*}}-\mathbb{E}_{\mathcal{A}}\right\|_{\infty, 2} \leq 4\left\|u-\mathbb{E}_{\mathcal{A}}(u)\right\|_{2}
$$

holds for all masas, [10]. In the same paper, we introduced the notion of an asymptotic homomorphism. This is the conditional expectation $\mathbb{E}_{\mathcal{A}}$ onto an abelian subalgebra $\mathcal{A}$ for which a unitary $u \in \mathcal{A}$ may be found to satisfy

$$
\lim _{|k| \rightarrow \infty}\left\|\mathbb{E}_{\mathcal{A}}\left(x u^{k} y\right)-\mathbb{E}_{\mathcal{A}}(x) \mathbb{E}_{\mathcal{A}}(y) u^{k}\right\|_{2}=0
$$

for all $x, y \in \mathcal{M}$. The masa associated to a generator in the von Neumann algebra of a free group is one particular example of this phenomenon, [10].

The above are all rigidity conditions on the abelian von Neumann subalgebra $\mathcal{A}$ of $\mathcal{M}$. The main point for asymptotic homomorphisms is that their existence implies both singularity and strong singularity, and that $\delta(\mathcal{A})=1$, [10, Theorem 4.7].

Let $\mathbb{F}_{k}$ denote the free group on $k$ generators, $2 \leq k<\infty$. We denote the generators of $\mathbb{F}_{k}$ by $\left\{g_{i}\right\}_{i=1}^{k}$, and we let

$$
S_{k}=\left\{g_{1}, \ldots, g_{k}, g_{1}^{-1}, \ldots, g_{k}^{-1}\right\}
$$

with cardinality $\left|S_{k}\right|=2 k$. For $n \geq 0$, we define $w_{n}$ to be the sum of all reduced words in $\mathbb{F}_{k}$ of length $n$. Then, for $n \geq 2$,

$$
\begin{aligned}
w_{0} & =e \\
w_{1} & =\sum_{i=1}^{k} g_{i}+\sum_{i=1}^{k} g_{i}^{-1} \\
w_{1}^{2} & =w_{2}+2 k w_{0} \\
w_{1} w_{n} & =w_{n} w_{1}=w_{n+1}+(2 k-1) w_{n-1}
\end{aligned}
$$

are examples of relations among these sums. They also show that the unital algebra generated by $w_{1}$ contains each $w_{n}$, and that this algebra is the span of these elements. The words in $w_{n}$ are pairwise orthogonal with respect to the trace, and so a simple counting argument gives the well-known formula

$$
\left\|w_{n}\right\|_{2}^{2}=2 k(2 k-1)^{n-1}, \quad n \geq 1,
$$

since this is the number of words of length $n$.

Let $\mathcal{B}$ denote the abelian von Neumann algebra generated by $w_{1}$. Then $\mathcal{B}$ is a masa, 8 , and our previous remarks show that

$$
\left\{w_{n} /\left\|w_{n}\right\|_{2}: n \geq 0\right\}
$$

is an orthonormal basis for $L^{2}(\mathcal{B}, \operatorname{tr})$. Moreover, for each $x \in \mathcal{M}$,

$$
\mathbb{E}_{\mathcal{B}}(x)=\sum_{n=0}^{\infty} \operatorname{tr}\left(x w_{n}\right) w_{n} /\left\|w_{n}\right\|_{2}^{2}
$$

\section{AsYmptotic homomorphisms}

In this section we present a criterion for determining when a conditional expectation is an asymptotic homomorphism, phrased in terms of an orthonormal basis for $L^{2}(\mathcal{B}, \operatorname{tr})$. 
Theorem 3.1. Let $\mathcal{A}$ be an abelian von Neumann subalgebra of a type $\mathrm{II}_{1}$ factor $\mathcal{M}$, and suppose that there is a *-isomorphism $\pi: \mathcal{A} \rightarrow L^{\infty}[0,1]$ which induces an isometry from $L^{2}\left(\mathcal{A}\right.$, tr) onto $L^{2}[0,1]$. Let

$$
\left\{v_{n}: n \geq 0, \quad v_{n} \in \mathcal{A}\right\}
$$

be an orthonormal basis for $L^{2}(\mathcal{A}, \operatorname{tr})$, and let $Y \subseteq \mathcal{M}$ be a set whose linear span is norm dense in $L^{2}(\mathcal{M}$, tr $)$. If

$$
\sum_{n=0}^{\infty}\left\|\mathbb{E}_{\mathcal{A}}\left(x v_{n} y\right)-\mathbb{E}_{\mathcal{A}}(x) \mathbb{E}_{\mathcal{A}}(y) v_{n}\right\|_{2}^{2}<\infty
$$

for all $x, y \in Y$, then $\mathbb{E}_{\mathcal{A}}$ is an asymptotic homomorphism, $\mathcal{A}$ is a strongly singular masa, and $\delta(\mathcal{A})=1$.

Proof. Suppose that there is a unitary $u \in \mathcal{A}$ for which

$$
\lim _{|n| \rightarrow \infty}\left\|\mathbb{E}_{\mathcal{A}}\left(x u^{n} y\right)-\mathbb{E}_{\mathcal{A}}(x) \mathbb{E}_{\mathcal{A}}(y) u^{n}\right\|_{2}=0
$$

when $x$ and $y$ are arbitrary elements of $Y$. Then (3.2) will hold for $x, y \in \operatorname{span} Y$, and the $\|\cdot\|_{2}$-norm continuity of $\mathbb{E}_{\mathcal{A}}$ will extend its validity to all $x, y \in \mathcal{M}$. Thus we may restrict attention to $x, y \in Y$.

Let $w$ be the unitary $e^{2 \pi i t}, 0 \leq t \leq 1$, which generates $L^{\infty}[0,1]$, and let $u=$ $\pi^{-1}(w)$ be the corresponding unitary generator of $\mathcal{A}$. Since $\pi$ is an isometry for the Hilbert space norms, we have

$$
\operatorname{tr}\left(v_{j}^{*} u^{n}\right)=\left\langle u^{n}, v_{j}\right\rangle=\int_{0}^{1} e^{2 \pi i n t} \overline{\pi\left(v_{j}\right)(t)} d t .
$$

From this it follows that

$$
\lim _{|n| \rightarrow \infty}\left\langle u^{n}, v_{j}\right\rangle=0, \quad j \geq 0,
$$

because the right-hand side of (3.3.3) is the conjugate of the $n^{\text {th }}$ Fourier coefficient of $\pi\left(v_{j}\right) \in L^{2}[0,1]$.

Fix $x, y \in Y$, let $\varepsilon>0$, and choose $k$, by (3.1), so that

$$
\sum_{j>k}\left\|\mathbb{E}_{\mathcal{A}}\left(x v_{j} y\right)-\mathbb{E}_{\mathcal{A}}(x) \mathbb{E}_{\mathcal{A}}(y) v_{j}\right\|_{2}^{2}<\varepsilon^{2} .
$$

From (3.4), we may now choose $n_{0}$ so that

$$
\left\langle u^{n}, v_{j}\right\rangle \leq \varepsilon((k+1)\|x\|\|y\|)^{-1}, \quad 0 \leq j \leq k, \quad|n| \geq n_{0} .
$$

Since $\left\{v_{j}\right\}_{j=1}^{\infty}$ is an orthonormal basis, we may write $u^{n}=\sum_{j=0}^{\infty}\left\langle u^{n}, v_{j}\right\rangle v_{j}$, which gives

$$
\left\|\mathbb{E}_{\mathcal{A}}\left(x u^{n} y\right)-\mathbb{E}_{\mathcal{A}}(x) \mathbb{E}_{\mathcal{A}}(y) u^{n}\right\|_{2}=\left\|\sum_{j=0}^{\infty}\left\langle u^{n}, v_{j}\right\rangle\left(\mathbb{E}_{\mathcal{A}}\left(x v_{j} y\right)-\mathbb{E}_{\mathcal{A}}(x) \mathbb{E}_{\mathcal{A}}(y) v_{j}\right)\right\|_{2} .
$$


We split this sum at $k$ and estimate each part separately. By (3.6),

$$
\begin{aligned}
& \| \sum_{j=0}^{k}\left\langle u^{n}, v_{j}\right\rangle\left(\mathbb{E}_{\mathcal{A}}\left(x v_{j} y\right)-\mathbb{E}_{\mathcal{A}}(x) \mathbb{E}_{\mathcal{A}}(y) v_{j} \|_{2}\right. \\
& \quad \leq \sum_{j=0}^{k} \varepsilon((k+1)\|x\|\|y\|)^{-1}\left\|\mathbb{E}_{\mathcal{A}}\left(x v_{j} y\right)-\mathbb{E}_{\mathcal{A}}(x) \mathbb{E}_{\mathcal{A}}(y) v_{j}\right\|_{2} \\
& \quad \leq \sum_{j=0}^{k} \varepsilon((k+1)\|x\|\|y\|)^{-1} 2\|x\|\|y\| \\
& \quad=2 \varepsilon
\end{aligned}
$$

provided that $|n| \geq n_{0}$. Since

$$
1=\left\|u^{n}\right\|_{2}^{2}=\sum_{j=0}^{\infty}\left|\left\langle u^{n}, v_{j}\right\rangle\right|^{2},
$$

we may use the Cauchy-Schwarz inequality to estimate

$$
\begin{aligned}
& \| \sum_{j=k+1}^{\infty}\left\langle u^{n}, v_{j}\right\rangle\left(\mathbb{E}_{\mathcal{A}}\left(x v_{j} y\right)-\mathbb{E}_{\mathcal{A}}(x) \mathbb{E}_{\mathcal{A}}(y) v_{j} \|_{2}\right. \\
& \leq \sum_{j=k+1}^{\infty}\left|\left\langle u^{n}, v_{j}\right\rangle\right|\left\|\mathbb{E}_{\mathcal{A}}\left(x v_{j} y\right)-\mathbb{E}_{\mathcal{A}}(x) \mathbb{E}_{\mathcal{A}}(y) v_{j}\right\|_{2} \\
& \quad \leq\left(\sum_{j=k+1}^{\infty}\left\|\mathbb{E}_{\mathcal{A}}\left(x v_{j} y\right)-\mathbb{E}_{\mathcal{A}}(x) \mathbb{E}_{\mathcal{A}}(y) v_{j}\right\|_{2}^{2}\right)^{1 / 2} \\
& \quad<\varepsilon
\end{aligned}
$$

by the choice of $k$ (see (3.5)). Then (3.8) and (3.10) allow us to obtain the inequality

$$
\left\|\mathbb{E}_{\mathcal{A}}\left(x u^{n} y\right)-\mathbb{E}_{\mathcal{A}}(x) \mathbb{E}_{\mathcal{A}}(y) u^{n}\right\|_{2}<3 \varepsilon, \quad|n| \geq n_{0},
$$

from (3.7). This proves (3.2), and the proof is complete.

\section{EnUmeration OF WORDS in $\mathbb{F}_{\boldsymbol{k}}$}

This section contains the main technical results which we will use to show that $\mathbb{E}_{\mathcal{B}}$ is an asymptotic homomorphism. We fix an integer $k \geq 2$, and formulate our results for $\mathbb{F}_{k}$. Some of the quantities below depend on $k$, but we have suppressed this for notational convenience.

Definition 4.1. Let $\left\{g_{1}, \ldots, g_{k}\right\}$ be the generators of $\mathbb{F}_{k}$ and let

$$
S_{k}=\left\{g_{1}, \ldots, g_{k}, g_{1}^{-1}, \ldots, g_{k}^{-1}\right\} .
$$

For $x, y \in S_{k}$, we let $w_{n}(x, y)$ be the sum of all words of length $n$ beginning with $x$ and ending with $y$, and we denote by $\nu_{n}(x, y)$ the number of such words. If $\sigma$ and $\tau$ are nonempty subsets of $S_{k}$, we write $\nu_{n}(\sigma, \tau)$ for the number of words of length $n$ which begin with an element of $\sigma$ and end with an element of $\tau$. 
We note that the formula

$$
\nu_{n}(\sigma, \tau)=\sum_{\substack{x \in \sigma \\ y \in \tau}} \nu_{n}(x, y)
$$

is an immediate consequence of these definitions, for any nonempty subsets $\sigma$ and $\tau$ of $S_{k}$. The following lemma is obvious, and we omit the proof.

Lemma 4.2. Let $\sigma=S_{k} \backslash\left\{g_{1}, g_{2}, g_{1}^{-1}, g_{2}^{-1}\right\}$ and $\tau=S_{k} \backslash\left\{g_{1}, g_{1}^{-1}\right\}$. Then, for $n \geq 2$,

$$
\begin{aligned}
& w_{n+1}\left(g_{1}, g_{2}\right)=g_{1}\left(w_{n}\left(g_{1}, g_{2}\right)+w_{n}\left(g_{2}, g_{2}\right)+w_{n}\left(g_{2}^{-1}, g_{2}\right)+\sum_{x \in \sigma} w_{n}\left(x, g_{2}\right)\right) \\
& w_{n+1}\left(g_{1}, g_{1}\right)=g_{1}\left(w_{n}\left(g_{1}, g_{1}\right)+\sum_{x \in \tau} w_{n}\left(x, g_{1}\right)\right)
\end{aligned}
$$

$$
w_{n+1}\left(g_{1}, g_{1}^{-1}\right)=g_{1}\left(w_{n}\left(g_{1}, g_{1}^{-1}\right)+\sum_{x \in \tau} w_{n}\left(x, g_{1}^{-1}\right)\right) .
$$

For $n \geq 2$, we introduce three constants $\alpha_{n}, \beta_{n}$, and $\gamma_{n}$ which are respectively $\nu_{n}\left(g_{1}, g_{2}\right), \nu_{n}\left(g_{1}, g_{1}\right)$ and $\nu_{n}\left(g_{1}, g_{1}^{-1}\right)$. For any pair $x, y \in S_{k}$, there is an automorphism of $\mathbb{F}_{k}$ which takes $w_{n}(x, y)$ to one of $w_{n}\left(g_{1}, g_{2}\right), w_{n}\left(g_{1}, g_{1}\right)$ and $w_{n}\left(g_{1}, g_{1}^{-1}\right)$, and $\nu_{n}(x, y)$ is $\alpha_{n}, \beta_{n}$ or $\gamma_{n}$.

Lemma 4.3. The following relations hold for $\alpha_{n}, \beta_{n}$ and $\gamma_{n}$ :

(i) For $n=2$,

$$
\alpha_{2}=1, \quad \beta_{2}=1, \quad \gamma_{2}=0 .
$$

(ii) For $n \geq 2$,

$$
\begin{aligned}
\alpha_{n+1} & =(2 k-3) \alpha_{n}+\beta_{n}+\gamma_{n}, \\
\beta_{n+1} & =\beta_{n}+(2 k-2) \alpha_{n}, \\
\gamma_{n+1} & =\gamma_{n}+(2 k-2) \alpha_{n} .
\end{aligned}
$$

(iii) There exists a constant $C_{k}$ such that, for $n \geq 2$,

(4.9) $\left|\alpha_{n}-(2 k-1)^{n-1} / 2 k\right|,\left|\beta_{n}-(2 k-1)^{n-1} / 2 k\right|,\left|\gamma_{n}-(2 k-1)^{n-1} / 2 k\right| \leq C_{k}$.

Proof. (i) Clear.

(ii) These are the result of counting the terms in (4.2), (4.3) and (4.4) and noting that the cardinalities of $\sigma$ and $\tau$ in Lemma 4.2 are respectively $2 k-4$ and $2 k-2$.

(iii) Equations (4.7) and (4.8) show that

$$
\beta_{n+1}-\gamma_{n+1}=\beta_{n}-\gamma_{n},
$$

and so these differences are independent of $n$. Since $\beta_{2}-\gamma_{2}=1$, we conclude that

$$
\beta_{n}=1+\gamma_{n}, \quad n \geq 2 .
$$

Subtraction of (4.8) from (4.6) gives

$$
\alpha_{n+1}-\gamma_{n+1}=\beta_{n}-\alpha_{n}=1+\left(\gamma_{n}-\alpha_{n}\right),
$$


using (4.11). Since $\alpha_{2}-\gamma_{2}=1$, a simple induction argument, based on (4.12), shows that

$$
\alpha_{n}=\gamma_{n}+\left(1+(-1)^{n}\right) / 2, \quad n \geq 2 .
$$

It follows from (4.11) and (4.13) that

$$
\left|\alpha_{n}-\gamma_{n}\right| \leq 1, \quad\left|\alpha_{n}-\beta_{n}\right| \leq 2, \quad n \geq 2 .
$$

There are $2 k(2 k-1)^{n-1}$ words in $w_{n}$. So counting these words according to whether they lie in a sum of the form $w_{n}(a, b), w_{n}(a, a)$ or $w_{n}\left(a, a^{-1}\right)$ leads to

$$
2 k(2 k-2) \alpha_{n}+2 k \beta_{n}+2 k \gamma_{n}=2 k(2 k-1)^{n-1},
$$

which, after cancellation, is

$$
(2 k-2) \alpha_{n}+\beta_{n}+\gamma_{n}=(2 k-1)^{n-1} .
$$

Substituting from (4.14) gives

$$
\left|2 k \alpha_{n}-(2 k-1)^{n-1}\right| \leq 3 .
$$

Now (4.9) follows from (4.17) and (4.14) if we define $C_{k}$ to be $2+3 /(2 k)$.

Remark 4.4. As is surely well known, equations (4.6)-(4.8) give closed form expressions for $\alpha_{n}, \beta_{n}$ and $\gamma_{n}$. One way to see this is to rewrite these equations as $\xi_{n+1}=A \xi_{n}$, with solution $\xi_{n}=A^{n-2} \xi_{2}$, where

$$
\xi_{n}=\left(\begin{array}{c}
\alpha_{n} \\
\beta_{n} \\
\gamma_{n}
\end{array}\right), \quad A=\left(\begin{array}{ccc}
2 k-3 & 1 & 1 \\
2 k-2 & 1 & 0 \\
2 k-2 & 0 & 1
\end{array}\right) .
$$

Explicit solutions can then be exhibited by observing that the eigenvalues of $A$ are $2 k-1,1$ and -1 , with respective eigenvectors

$$
(1,1,1)^{\mathrm{T}}, \quad(0,1,-1)^{\mathrm{T}}, \quad(-1, k-1, k-1)^{\mathrm{T}} .
$$

We omit the details, noting that it is more convenient for us to work subsequently with the inexact estimates of (4.9) than with the exact expressions thus obtained.

Corollary 4.5. There exists a constant $D_{k}$, depending only on $k$, such that

$$
\left|\nu_{n}\left(\sigma_{1}, \tau_{1}\right)-\nu_{n}\left(\sigma_{2}, \tau_{2}\right)\right| \leq D_{k}
$$

whenever $\sigma_{1}, \sigma_{2}, \tau_{1}, \tau_{2}$ are nonempty subsets of $S_{k}$ satisfying

$$
\left|\sigma_{1}\right|=\left|\sigma_{2}\right|, \quad\left|\tau_{1}\right|=\left|\tau_{2}\right| .
$$

Proof. First suppose that these are one-point sets. Then each $\nu_{n}(\cdot, \cdot)$ is either $\alpha_{n}, \beta_{n}$ or $\gamma_{n}$; so the difference is estimated by $2 C_{k}$, using (4.9). For the general case, (4.1) shows that this difference can be realized as a sum of $\left|\sigma_{1}\right|\left|\tau_{1}\right|$ differences for one-point sets. This gives the estimate

$$
\left|\nu_{n}\left(\sigma_{1}, \tau_{1}\right)-\nu_{n}\left(\sigma_{2}, \tau_{2}\right)\right| \leq 2\left|\sigma_{1}\right|\left|\tau_{1}\right| C_{k} .
$$

Let $D_{k}$ be the largest possible right-hand side in (4.20), which is $8 k^{2} C_{k}$.

Lemma 4.6. Let $x=x_{\ell} \ldots x_{1}$ and $y=y_{1} \ldots y_{m}$ be words in $\mathbb{F}_{k}$, and let $\mu(r, s, n$; $x, y)$ be the number of reduced words in the product $x w_{n} y$ which result from $r$ cancellations on the left and s cancellations on the right. Then there exist subsets $\sigma_{r}(x)$, $\tau_{s}(y)$ of $S_{k}$, whose cardinalities depend only on $r$ and $s$ respectively, such that

$$
\mu(r, s, n ; x, y)=\nu_{n-r-s}\left(\sigma_{r}(x), \tau_{s}(y)\right),
$$


for $n \geq \ell+m+2,0 \leq r \leq \ell, 0 \leq s \leq m$.

Proof. We first define $\sigma_{r}(x)$ and $\tau_{s}(y)$, for $0 \leq r \leq \ell, 0 \leq s \leq m$, and then verify that they have the required properties. Let

$$
\sigma_{0}(x)=S_{k} \backslash\left\{x_{1}^{-1}\right\}, \sigma_{\ell}(x)=S_{k} \backslash\left\{x_{\ell}\right\}, \tau_{0}(y)=S_{k} \backslash\left\{y_{1}^{-1}\right\}, \tau_{m}(y)=S_{k} \backslash\left\{y_{m}\right\} .
$$

For $0<r<\ell$ and $0<s<m$, let

$$
\sigma_{r}(x)=S_{k} \backslash\left\{x_{r+1}^{-1}, x_{r}\right\}, \tau_{s}(y)=S_{k} \backslash\left\{y_{s+1}^{-1}, y_{s}\right\} .
$$

Note that the pairs $\left\{x_{r+1}^{-1}, x_{r}\right\}$ and $\left\{y_{s+1}^{-1}, y_{s}\right\}$ are distinct, since otherwise cancellations would occur in $x$ or $y$. Thus the cardinalities of the sets in (4.22) and (4.23) are respectively $2 k-1$ and $2 k-2$, and they depend only on $r$ and $s$. If the reduced word

$$
x_{\ell} \ldots x_{r+1} v y_{s+1} \ldots y_{m}
$$

results from one of these cancellations, then the first letter of $v$ must not be $x_{r+1}^{-1}$ (if $r=\ell$, then this constraint disappears). The original reduced word in $w_{n}$ which canceled to this was

$$
x_{1}^{-1} \ldots x_{r}^{-1} v y_{s}^{-1} \ldots y_{1}^{-1}
$$

which requires the first letter of $v$ to be different from $x_{r}$ (this constraint disappears for $r=0)$. Thus the first letter of $v$ must lie in $\sigma_{r}(x)$. Conversely, such a $v$ allows exactly $r$ cancellations on the left in (4.25). A similar analysis on the right shows that a word of the form (4.25) both is reduced and allows the correct number of cancellations precisely when $v$ starts with an element of $\sigma_{r}(x)$ and ends with an element of $\tau_{s}(y)$. This proves the result.

\section{Asymptotic homomorphisms}

We now apply the results of the previous section to show that $\mathbb{E}_{\mathcal{B}}$ is an asymptotic homomorphism. In order to use Theorem 3.1 in Theorems 5.1 and 5.4, we need to know that the spectrum of $w_{1}$ is a closed interval, which may be found in [3], [8]. The omission of this point in an earlier version of the paper was kindly brought to our attention by Stuart White.

Theorem 5.1. Let $k \geq 2$ and let $\mathcal{B}$ be the Laplacian masa in $V N\left(\mathbb{F}_{k}\right)$. Then $\mathbb{E}_{\mathcal{B}}$ is an asymptotic homomorphism.

Proof. Let $x$ and $y$ in $\mathbb{F}_{k}$ be fixed words of lengths $\ell$ and $m$ respectively. We require that $\ell, m \geq 1$, since otherwise the inequality (5.10), for which we are aiming, is trivial. Let $z$ be an arbitrary word of length $\ell$, and suppose throughout that $n \geq \ell+m+2$.

If a word $v$ has length $p$, then it is orthogonal to $w_{n}$ for $n \neq p$. From this and (2.7), it follows that

$$
\mathbb{E}_{\mathcal{B}}(v)=w_{p}\left\|w_{p}\right\|_{2}^{-2}
$$

Thus, with the notation of Lemma 4.6.

$$
\mathbb{E}_{\mathcal{B}}\left(x w_{n} y\right)=\sum_{r=0}^{\ell} \sum_{s=0}^{m} \nu_{n-r-s}\left(\sigma_{r}(x), \tau_{s}(y)\right) w_{n+\ell+m-2(r+s)}\left\|w_{n+\ell+m-2(r+s)}\right\|_{2}^{-2} .
$$


By Corollary 4.5 and Lemma 4.6,

$$
\left|\nu_{p}\left(\sigma_{r}(x), \tau_{s}(y)\right)-\nu_{p}\left(\sigma_{r}(z), \tau_{s}(y)\right)\right| \leq D_{k}
$$

for $p \geq 2$. Thus there exist constants $\lambda_{r, s}$, uniformly bounded by $D_{k}$, such that

$$
\mathbb{E}_{\mathcal{B}}\left(x w_{n} y\right)-\mathbb{E}_{\mathcal{B}}\left(z w_{n} y\right)=\sum_{r=0}^{\ell} \sum_{s=0}^{m} \lambda_{r, s} w_{n+\ell+m-2(r+s)}\left\|w_{n+\ell+m-2(r+s)}\right\|_{2}^{-2} .
$$

Now $\left\|w_{p}\right\|_{2}^{2}$ is the number of terms in $w_{p}$; so

$$
\left\|w_{p}\right\|_{2}^{2}=2 k(2 k-1)^{p-1}=(2 k-1)^{p-n}\left\|w_{n}\right\|_{2}^{2} .
$$

Thus

$$
\left\|w_{n+\ell+m-2(r+s)}\right\|_{2}^{2}=(2 k-1)^{\ell+m-2(r+s)}\left\|w_{n}\right\|_{2}^{2}
$$

$\mathrm{SO}$

$$
\left\|w_{n+\ell+m-2(r+s)}\right\|_{2}^{2} \geq(2 k-1)^{-(\ell+m)}\left\|w_{n}\right\|_{2}^{2},
$$

for $0 \leq r \leq \ell, 0 \leq s \leq m$. This last inequality and (5.4) then give the estimate

$$
\left\|\mathbb{E}_{\mathcal{B}}\left(x w_{n} y\right)-\mathbb{E}_{\mathcal{B}}\left(z w_{n} y\right)\right\|_{2} \leq(\ell+1)(m+1) D_{k}(2 k-1)^{(\ell+m) / 2}\left\|w_{n}\right\|_{2}^{-1} .
$$

Let $H_{\ell, m, k}$ be the constant on the right-hand side of (5.8). If we sum (5.8) over all words $z$ of length $\ell$ (of which there are $\left\|w_{\ell}\right\|_{2}^{2}$ ), we obtain

$$
\|\| w_{\ell}\left\|_{2}^{2} \mathbb{E}_{\mathcal{B}}\left(x w_{n} y\right)-\mathbb{E}_{\mathcal{B}}\left(w_{\ell} w_{n} y\right)\right\|_{2} \leq\left\|w_{\ell}\right\|_{2}^{2} H_{\ell, m, k}\left\|w_{n}\right\|_{2}^{-1} .
$$

Since $\mathbb{E}_{\mathcal{B}}(x)=w_{\ell}\left\|w_{\ell}\right\|_{2}^{-2}$ and $\mathbb{E}_{\mathcal{B}}\left(w_{\ell} w_{n} y\right)=w_{\ell} w_{n} \mathbb{E}_{\mathcal{B}}(y)$, (5.9) implies that

$$
\left\|\mathbb{E}_{\mathcal{B}}\left(x w_{n} y\right)-\mathbb{E}_{\mathcal{B}}(x) \mathbb{E}_{\mathcal{B}}(y) w_{n}\right\|_{2} \leq H_{\ell, m, k}\left\|w_{n}\right\|_{2}^{-1} .
$$

If we let $v_{n}=w_{n}\left\|w_{n}\right\|_{2}^{-1}$, then the terms of the series in (3.1) are bounded by $H_{\ell, n, k}^{2}\left\|w_{n}\right\|_{2}^{-4}$, and the series is clearly summable. The result now follows from Theorem 3.1 .

Theorem 5.2. Fix $k \geq 2$. Then the Laplacian masa $\mathcal{B}$ in $\mathbb{F}_{k}$ is strongly singular, and has Popa invariant $\delta(\mathcal{B})=1$.

Proof. Apply Theorem 5.1 and [10, Theorem 4.7].

Remark 5.3. Theorem 5.2 gives a new proof that $\mathcal{B}$ is a singular masa, a result originally due to Rădulescu, 9.

We now extend our results to the free product of a finite number of countable discrete groups $G_{i}, 1 \leq i \leq m, m \geq 2$. We let $G$ denote $G_{1} * G_{2} * \ldots * G_{m}$, and we fix elements $g_{i} \in G_{i}, 1 \leq i \leq k$. We assume that each $g_{i}$ has infinite order, which is possible in many, but not all, such groups. We denote by $\mathcal{B}$ the abelian subalgebra of $V N(G)$ generated by the selfadjoint element

$$
h=\sum_{i=1}^{k}\left(g_{i}+g_{i}^{-1}\right) .
$$

Theorem 5.4. With the above notation, the conditional expectation $\mathbb{E}_{\mathcal{B}}$ of $V N(G)$ onto $\mathcal{B}$ is an asymptotic homomorphism, $\mathcal{B}$ is a strongly singular masa, and $\delta(\mathcal{B})=$ 1. 
Proof. The elements $g_{1}, \ldots, g_{k}$ generate a copy of $\mathbb{F}_{k}$ inside $G$, and we write $\mathcal{N}$ for the resulting subfactor of $V N(G)$. As before, we let $w_{n}$ be the sum of all words of length $n$ in $\mathbb{F}_{k}$, and we note that $\left\{v_{n}=w_{n} /\left\|w_{n}\right\|_{2}\right\}_{n=0}^{\infty}$ is an orthonormal basis for $L^{2}(\mathcal{B})$. The result will follow from Theorem 3.1 if we can show that

$$
\sum_{n=0}^{\infty}\left\|\mathbb{E}_{\mathcal{B}}\left(x v_{n} y\right)-\mathbb{E}_{\mathcal{B}}(x) \mathbb{E}_{\mathcal{B}}(y) v_{n}\right\|_{2}^{2}<\infty
$$

for all $x, y \in G$.

If $x \in \mathbb{F}_{k}, y \in G$, then

$$
\mathbb{E}_{\mathcal{B}}\left(x v_{n} y\right)=\mathbb{E}_{\mathcal{B}}\left(\mathbb{E}_{\mathcal{N}}\left(x v_{n} y\right)\right)=\mathbb{E}_{\mathcal{B}}\left(x v_{n} \mathbb{E}_{\mathcal{N}}(y)\right),
$$

using modularity of $\mathbb{E}_{\mathcal{N}}$. If $y \in G \backslash \mathbb{F}_{k}$, then $\mathbb{E}_{\mathcal{N}}(y)=0$; so all terms in (5.11) vanish. If $y \in \mathbb{F}_{k}$, then the argument of Theorem 5.1 shows the validity of (5.11). A similar analysis holds if we begin by supposing that $y \in \mathbb{F}_{k}$. We may thus assume that $x, y \in G \backslash \mathbb{F}_{k}$. In this case $\mathbb{E}_{\mathcal{B}}(x)=0$; so (5.11) becomes

$$
\sum_{n=0}^{\infty}\left\|\mathbb{E}_{\mathcal{B}}\left(x v_{n} y\right)\right\|_{2}^{2}<\infty .
$$

The elements $x$ and $y$ may be written as products

$$
x=x_{\ell} \ldots x_{1}, \quad y=y_{1} \ldots y_{q},
$$

where $x_{i} \in G_{s_{i}}, y_{j} \in G_{t_{j}}$, and $s_{i} \neq s_{i+1}, t_{j} \neq t_{j+1}$. Let

$$
\chi_{n}=\left\{u \in \mathbb{F}_{k}:|u|=n, \text { xuy } \in \mathbb{F}_{k}\right\},
$$

where $|u|$ is the length of $u$ in terms of $g_{1}, \ldots, g_{k} \in \mathbb{F}_{k}$. We will show that the cardinality $\left|\chi_{n}\right|$ is at most $(2 n+1)(n+1)$. Consider a word $u \in \mathbb{F}_{k}$ of length $n$ and let

$$
u=u_{1} \ldots u_{r}
$$

be written in the free product $G$ with each $u_{j}$ a nonzero power of some $g_{i_{j}}$, and $i_{j} \neq i_{j+1}$ for all $j$. Note that $r \leq n$. We observe that the product $x u y$ is in $\mathbb{F}_{k}$ if and only if one of the following conditions is satisfied:

(1) there exists $p, 0 \leq p \leq r$, such that $u_{i}=x_{i}^{-1}$ for $1 \leq i \leq p$, and $u_{r-i+1}=y_{i}^{-1}$ for $1 \leq i \leq r-p$ (with obvious modifications if $p=0$ or $p=r$ ), and the element

$$
x u y=x_{\ell} \ldots x_{p+1} y_{r-p+1} \ldots y_{q}
$$

is in $\mathbb{F}_{k}$;

(2) there exists $p, 0 \leq p \leq r$, such that $u_{i}=x_{i}^{-1}$ for $1 \leq i \leq p-1$, and $u_{r-i+1}=y_{i}^{-1}$ for $1 \leq i \leq r-p$ (with obvious modifications if $p=0$ or $p=r$ ), and the element

$$
x u y=x_{\ell} \ldots x_{p} u_{p} y_{r-p+1} \ldots y_{q}
$$

is in $\mathbb{F}_{k}$.

In (5.15), $x_{p+1}$ and $y_{r-p+1}$ are in the same $G_{i}$, since further cancellation must occur, while in (5.16) the same conclusion applies to the elements $x_{p}, u_{p}$, and $y_{r-p+1}$. In (1), there are at most $n+1$ values of $p$, and thus at most the same number of possibilities for $u$. In (2), $p$ takes up to $n+1$ values, and the remaining term $u_{p}$ is a nonzero power $g_{i}^{s}$ for some $i$ and some $s$ with $1 \leq|s| \leq n$. This gives an upper bound of $2 n(n+1)$ possibilities. The two cases combine to give an 
upper estimate (which could undoubtedly be lowered by a more detailed analysis) of $(n+1)(2 n+1)$ for $\left|\chi_{n}\right|$.

It now follows that

$$
\left\|\mathbb{E}_{\mathcal{B}}\left(x w_{n} y\right)\right\|_{2} \leq\left|\chi_{n}\right| \leq(n+1)(2 n+1),
$$

since $\| \mathbb{E}_{\mathcal{B}}($ xuy $) \|_{2} \leq 1$ for each $u \in \chi_{n}$. Thus the terms in (5.13) are dominated by $(n+1)^{2}(2 n+1)^{2}\left\|w_{n}\right\|_{2}^{-2}$; so convergence is guaranteed by (2.6). This proves that $\mathbb{E}_{\mathcal{B}}$ is an asymptotic homomorphism, and the other statements now follow from [10]. Theorem 4.7].

Remark 5.5. The conclusions of Theorem 5.4 would remain valid were we to replace each $g_{i}$ by some nonzero power $g_{i}^{t_{i}}$, and $h$ by

$$
\sum_{i=1}^{k}\left(g_{i}^{t_{i}}+g_{i}^{-t_{i}}\right)
$$

since $\left\{g_{i}^{t_{i}}\right\}_{i=1}^{k}$ also generates a copy of $\mathbb{F}_{k}$ in $G$.

\section{REFERENCES}

[1] F. Boca and F. Rădulescu, Singularity of radial subalgebras in $\mathrm{II}_{1}$ factors associated with free products of groups, J. Funct. Anal., 103 (1992), 138-159. MR 93d:46103

[2] J. Dixmier, Sous-anneaux abéliens maximaux dans les facteurs de type fini, Ann. Math., 59 (1954), 279-286. MR 15:539b

[3] A. Figà-Talamanca and M. Picardello, Harmonic analysis on free groups, Lecture Notes in Pure and Applied Mathematics, 87, Marcel Dekker, New York, 1983. MR 85j:43001

[4] S. Popa, Singular maximal abelian $*$-subalgebras in continuous von Neumann algebras, J. Funct. Anal., 50 (1983), 151-166. MR 84e:46065

[5] S. Popa, Notes on Cartan subalgebras in type $\mathrm{II}_{1}$ factors, Math. Scand., 57 (1985), 171-188. MR 87f:46114

[6] S. Popa, On the distance between masas in type $\mathrm{II}_{1}$ factors, Math. Phys. in Math. and Phys. (Siena, 2000), Fields Inst. Commun., vol. 30, Amer. Math. Soc., Providence, RI, 2001, pp. 321-324.

[7] L. Pukánszky, On maximal abelian subrings of factors of type $\mathrm{II}_{1}$, Canad. J. Math., 12 (1960), 289-296. MR 22:3992

[8] T. Pytlik, Radial functions on free groups and a decomposition of the regular representation into irreducible components, J. Reine Angew. Math., 326 (1981), 124-135. MR 84a:22017

[9] F. Rădulescu, Singularity of the radial subalgebra of $\mathcal{L}\left(F_{N}\right)$ and the Pukánszky invariant, Pacific J. Math., 151 (1991), 297-306. MR 93b:46120

[10] A. M. Sinclair and R. R. Smith, Strongly singular masas in type $\mathrm{II}_{1}$ factors, Geom. Funct. Anal., 12 (2002), 199-216.

[11] D. Voiculescu, The analogues of entropy and of Fisher's information measure in free probability theory. III. The absence of Cartan subalgebras, Geom. Funct. Anal., 6 (1996), 172-199. MR 96m:46119

School of Mathematics, University of Edinburgh, Edinburgh EH9 3JZ, Scotland

E-mail address: allan@maths.ed.ac.uk

Department of Mathematics, Texas A\&M University, College Station, Texas 77843

E-mail address: rsmith@math.tamu.edu 